\title{
Calculation of Thermal State of Bodies with Multilayer Coatings
}

\author{
V. A. Shevchuk \\ Pidstryhach Institute for Applied Problems of Mechanics and Mathematics, \\ National Academy of Sciences of Ukraine, \\ 3b Naukova, Lviv, 79053, Ukraine \\ e-mail: shevchuk@iapmm.lviv.ua
}

\begin{abstract}
The procedure for the calculation of heat conduction process in constructions elements with thin protective multilayer coatings is elaborated. This procedure is based on the essential simplification of solving the initial problem for constructions elements with thin multilayer coatings, and is connected with the modeling of such coatings by thin shells with appropriate geometrical and thermal properties of a coating. In such an approach, the influence of thin coatings on the course of heat processes in a body-coating system is described by special generalized boundary conditions. The efficiency of this approach has been shown by the comparison of results obtained according to this approximate approach with an accurate solution for the test case of an n-layer plate.
\end{abstract}

\section{Introduction}

The bodies with thin multilayer coatings represent an important class of nonhomogeneous bodies. In practice to protect construction elements from the aggressive influence of environment, special coatings are used. It is essential that such coatings usually have non-homogeneous properties (which is connected with production conditions or caused by functional requirements) - multilayer coatings, in particular.

Several authors have investigated different heat conduction problems for these objects $[2,11,12]$. As a rule, such calculation for bodies with composite coatings is connected with formulating and solving appropriate problems of mathematical physics for multilayer systems, which are cumbersome and ineffective for practical purposes and are usually used as standards in elaborating approximate methods. Furthermore, the specific feature of such compound systems as bodies with composite coatings is the smallness of coating thickness in comparison with that of a substrate. Even numerical analysis of such compound structures, which contain substructures of different dimensionality, encounters significant difficulties [9]. It is natural, therefore, to attempt to build up approximate solutions, which are satisfactorily accurate for practical purposes. There have been suggested various approaches $[2,8,9,12]$ that allow us to take into account the smallness of coating thickness during the calculation of heat processes in the bodies with coatings. One of effective approaches consists in modeling of the influence of thin-walled elements of constructions by special boundary conditions $[1,3,5,6,10]$. This approach essentially simplifies solving the problems of finding the thermal state of constructions with thin multilayer coatings. 
It is based on the modeling of the coatings by thin shells with appropriate geometrical and thermal properties of a coating.

The elaboration of such generalized boundary conditions can be derived by different techniques. Depending on the type of boundary conditions, the kinds of considered non-linearities and the possibility of preliminary linearization, the following methods can be used:

(i) operator method, which allows us not to accept preliminary hypotheses of the distribution of desired functions over the thickness of coating layers [4, 7];

(ii) approach, based on the application of a priori assumptions of the distribution of desired functions over the thickness of coating layers or on the whole coating [6]; (iii) discrete approach, based on the appropriate difference approximations of normal derivatives in appropriate expansions [3].

In this article, the generalized boundary condition is constructed by the application of operator method, and the efficiency of the approach is illustrated by the example of the solution of a test problem.

\section{Problem Statement}

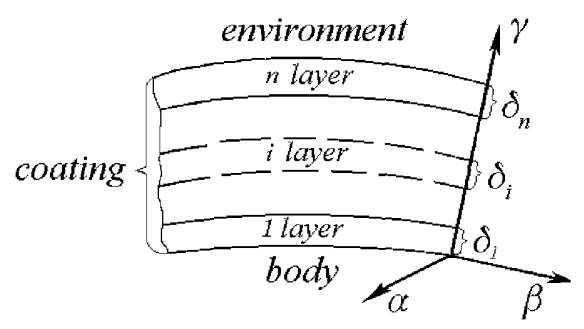

Fig. 1. Scheme of object

The object under investigation is a body with an applied thin multilayer coating with thickness $\delta=\sum_{i=1}^{n} \delta_{i}$, whose layers are made of different orthotropic materials. Here the $n$-layer coating is considered as a thin shell, referred to a mixed coordinate system $(\alpha, \beta, \gamma)$, which are respectively the lines of principal curvatures of the body-coating interface and the normal to it (Fig. 1). We assume that the edge surface $\Sigma$ of such a shell is a ruled surface, for which the contour $G$ bounding the body-coating interface $S$ is directrix, and the normal to $S$ in every point of the contour is generatrix.

We write the heat conduction equation for the each thin $i$ th layer as [7]

$$
\begin{gathered}
\lambda_{i}^{\gamma}\left(\frac{\partial^{2} t_{i}}{\partial \gamma^{2}}+2 k_{i} \frac{\partial t_{i}}{\partial \gamma}\right)+\Delta t_{i}=\omega_{i} \frac{\partial t_{i}}{\partial \tau} \\
\Delta_{i}=\frac{1}{A_{i} B_{i}}\left[\lambda_{i}^{\alpha} \frac{\partial}{\partial \alpha}\left(\frac{B_{i}}{A_{i}} \frac{\partial}{\partial \alpha}\right)+\lambda_{i}^{\beta} \frac{\partial}{\partial \beta}\left(\frac{A_{i}}{B_{i}} \frac{\partial}{\partial \beta}\right)\right], \quad k_{i}=\frac{k_{1 i}+k_{2 i}}{2}, \quad i=\overline{1, n} .
\end{gathered}
$$


We assume that the coating is heated by environment according to Newton's law

$$
\begin{gathered}
\lambda_{n}^{\gamma} \frac{\partial t_{n}}{\partial \gamma}=\mu\left(t_{e}-t_{n}\right) \quad \text { at } \gamma=\gamma_{n}=\sum_{i=1}^{n} \delta_{i}, \\
\mathrm{~L}_{i} t_{i}=\mu_{i}\left(t_{e}-t_{i}\right) \text { on } \Sigma, \mathrm{L}_{i}=n_{1} \frac{\lambda_{i}^{\alpha}}{A_{i}} \frac{\partial}{\partial \alpha}+n_{2} \frac{\lambda_{i}^{\beta}}{B_{i}} \frac{\partial}{\partial \beta}, i=\overline{1, n} ;
\end{gathered}
$$

and the ideal thermal contact occurs between the constituents of the coating and the body

$$
\begin{aligned}
& t_{i}=t_{i-1}, \quad \lambda_{i}^{\gamma} \frac{\partial t_{i}}{\partial \gamma}=\lambda_{i-1}^{\gamma} \frac{\partial t_{i-1}}{\partial \gamma} \text { at } \gamma=\gamma_{i-1}=\sum_{j=1}^{i-1} \delta_{j}, i=\overline{2, n} \\
& t_{1}=t_{b}, \quad \lambda_{1}^{\gamma} \frac{\partial t_{1}}{\partial \gamma}=\lambda_{b}^{\gamma} \frac{\partial t_{b}}{\partial \gamma} \text { at } \gamma=\gamma_{0}=0,
\end{aligned}
$$

and initial conditions are

$$
t_{i \mid \tau=0}=t_{i}^{0}(\alpha, \beta, \gamma) \quad i=\overline{1, n} .
$$

Here $t_{i}, t_{b}, t_{e}$ are the temperatures of the $i$ th coating layer, the body and the environment respectively; $A_{i}, B_{i}$ are the coefficients of the first fundamental form of the interface between the $i$ th and $(i-1)$ th layers $(i=\overline{2, n})$ and of the body-coating interface $(i=1) ; k_{1}, k_{2 i}$ are the curvatures of coordinate lines; $\delta_{i}$ is the thickness of the $i$ th layer; $\lambda_{i}^{\alpha}, \lambda_{i}^{\beta}, \lambda_{i}^{\gamma}$, and $\omega_{i}$ are the coefficients of heat conductivity and heat capacity; $\mu, \mu_{i}$ are the coefficients of heat output from coating surface $\gamma=\gamma_{n}$ and from $i$ th part of the edge surface $\Sigma ; n_{1}, n_{2}$ are the components of vector of outer normal to the edge surface $\Sigma$. The subscripts $i, b$, and $e$ refer to the $i$ th layer of the coating, the body, and the environment respectively.

\section{Derivation of Generalized Boundary Conditions}

Instead of the temperature $t_{i}$ we introduce the variable $[4,7]$

$$
\theta_{i}=\exp \left(k_{i} \gamma\right) t_{i} \quad i=\overline{1, n} .
$$

For its determination, we obtain the equation

$$
p_{i}^{2} \theta_{i}+\frac{\partial^{2} \theta_{i}}{\partial \gamma^{2}}=0, \quad \gamma \in\left(\gamma_{\mathrm{i}-1}, \gamma_{i}\right) i=\overline{1, n} ; \quad p_{i}^{2}=\frac{1}{\lambda_{i}^{\gamma}} \Delta_{\mathrm{i}}-\frac{\omega_{\mathrm{i}}}{\lambda_{i}^{\gamma}} \frac{\partial}{\partial \tau}-k_{i}^{2},
$$

and boundary and initial conditions 


$$
\begin{gathered}
\lambda_{n}^{\gamma}\left(\frac{\partial \theta_{n}}{\partial \gamma}-k_{n} \theta_{n}\right)=\mu\left[\exp \left(k_{n} \gamma_{n}\right) t_{e}-\theta_{n}\right] \quad \text { at } \gamma=\gamma_{n}, \\
\mathrm{~L}_{i}\left(\theta_{i}\right)=\mu_{i}\left[\left(\exp \left(k_{i} \gamma\right) t_{e}-\theta_{i}\right] \quad \text { on } \Sigma, \quad i=\overline{1, n}\right. \\
\lambda_{i}^{\gamma}\left(\frac{\partial \theta_{i}}{\partial \gamma}-k_{i} \theta_{i}\right)=\exp \left[\left(k_{i}-k_{i-1}\right) \gamma_{i-1}\right] \lambda_{i-1}^{\gamma}\left(\frac{\partial \theta_{i-1}}{\partial \gamma}-k_{i-1} \theta_{i-1}\right), \\
\theta_{i}=\exp \left[\left(k_{i}-k_{i-1}\right) \gamma_{i-1}\right] \theta_{i-1}, \quad \text { at } \gamma=\gamma_{i-1}, \quad i=\overline{2, n} \\
\lambda_{1}^{\gamma}\left(\frac{\partial \theta_{1}}{\partial \gamma}-k_{1} \theta_{1}\right)=\lambda_{b}^{\gamma}\left(\frac{\partial t}{\partial \gamma}\right)_{b}, \quad \theta_{1}=t_{b} \text { at } \gamma=\gamma_{0}, \\
\theta_{i \mid \tau=0}=\exp \left(k_{i} \gamma\right) t_{i}^{0} \quad i=\overline{1, n} .
\end{gathered}
$$

Let us introduce notations for the boundary values of the function $\theta_{i}$ and its derivatives:

$$
\begin{aligned}
& \theta_{i}^{+}=\lim _{\gamma \rightarrow \gamma_{i}-0} \theta_{i}, \quad\left(\frac{\partial \theta_{i}}{\partial \gamma}\right)^{+}=\lim _{\gamma \rightarrow \gamma_{i}-0}\left(\frac{\partial \theta_{i}}{\partial \gamma}\right), \\
& \theta_{i}^{-}=\lim _{\gamma \rightarrow \gamma_{i-1}+0} \theta_{i}, \quad\left(\frac{\partial \theta_{i}}{\partial \gamma}\right)^{-}=\lim _{\gamma \rightarrow \gamma_{i-1}+0}\left(\frac{\partial \theta_{i}}{\partial \gamma}\right) .
\end{aligned}
$$

Using the operator method [4, 7], we represent the general solution of Eq. (7) as the solution of an ordinary differential equation

$$
\theta_{i}=\frac{\theta_{i}^{-} \sin \left(p_{i}\left(\gamma_{i}-\gamma\right)\right)+\theta_{i}^{+} \sin \left(p_{i}\left(\gamma-\gamma_{i-1}\right)\right)}{\sin \left(p_{i} \delta_{i}\right)}, \quad i=\overline{1, n} .
$$

Substituting (14) into (10)-(12), we obtain the following recursive relations for the temperature and its derivative at the interfaces of neighboring layers

$$
\tilde{\theta}_{1}=\mathrm{D}_{1} \tilde{t}_{b}, \quad \tilde{\theta}_{i}=\mathrm{D}_{i} \tilde{\theta}_{i-1}, \quad i=\overline{2, n} ;
$$

where $\quad \tilde{\theta}_{i}=\left[\theta_{i}^{+},\left(\frac{\partial \theta_{i}}{\partial \gamma}\right)^{+}\right]^{\mathrm{T}}, i=\overline{1, n} ; \quad \tilde{t}_{b}=\left[t_{b},\left(\frac{\partial t}{\partial \gamma}\right)_{b}\right]^{\mathrm{T}}$,

$$
\mathrm{D}_{i}=\exp \left[\left(k_{i}-k_{i-1}\right) \gamma_{i-1}\right]\left[\begin{array}{ll}
\cos \left(p_{i} \delta_{i}\right)+\left(k_{i}-\frac{\lambda_{i-1}^{\gamma}}{\lambda_{i}^{\gamma}} k_{i-1}\right) \frac{\sin \left(p_{i} \delta_{i}\right)}{p_{i}} & \frac{\lambda_{i-1}^{\gamma}}{\lambda_{i}^{\gamma}} \frac{\sin \left(p_{i} \delta_{i}\right)}{p_{i}} \\
-p_{i} \sin \left(p_{i} \delta_{i}\right)+\left(k_{i}-\frac{\lambda_{i-1}^{\gamma}}{\lambda_{i}^{\gamma}} k_{i-1}\right) \cos \left(p_{i} \delta_{i}\right) & \frac{\lambda_{i-1}^{\gamma}}{\lambda_{i}^{\gamma}} \cos \left(p_{i} \delta_{i}\right)
\end{array}\right],
$$


when using supplement auxiliary notations $k_{0}=0, \lambda_{0}^{\gamma}=\lambda_{\mathrm{b}}^{\gamma}$.

Now we write also the representation for the temperature and its derivative through the values of temperature and its derivative at the coating-body interface:

$$
\tilde{\theta}_{i}=\mathrm{F}_{i} \tilde{t}_{b}, \quad i=\overline{1, n} ;
$$

where

$$
\mathrm{F}_{i}=\left[\begin{array}{cc}
f_{i}^{11} & f_{i}^{12} \\
f_{i}^{21} & f_{i}^{22}
\end{array}\right] .
$$

From (16), (15) follows:

$$
\mathrm{F}_{1}=\mathrm{D}_{1}, \quad \mathrm{~F}_{i}=\mathrm{D}_{i} \mathrm{~F}_{i-1}, \quad i=\overline{2, n} .
$$

Substitution of (16) at $i=n$ into (8) leads finally after certain transformations to

$$
a_{n 1} t_{b}+a_{n 2}\left(\frac{\partial t}{\partial \gamma}\right)_{b}-\mu t_{e}=0 \text { on } S,
$$

where coefficients $a_{n}=\left[a_{n 1}, a_{n 2}\right]$ are determined by recursive relations

$$
\begin{array}{ll}
a_{01}=\left(\mu-\lambda_{n}^{\gamma} k_{n}\right) \exp \left(-k_{n} \gamma_{n}\right), & a_{02}=\lambda_{n}^{\gamma} \exp \left(-k_{n} \gamma_{n}\right), \\
a_{i}=a_{i-1} \mathrm{D}_{n-i+1} \quad i=\overline{1, n} . &
\end{array}
$$

Edge (9) and initial (13) conditions are satisfied by integrating

$$
\begin{gathered}
\sum_{i=1}^{n}\left(\mathrm{~L}_{i}+\mu_{i}\right) \int_{\gamma_{i-1}}^{\gamma_{i}} \exp \left(-k_{i} \gamma\right) \theta_{i} d \gamma-\sum_{i=1}^{n} \mu_{i} \int_{\gamma_{i-1}}^{\gamma_{i}} t_{e} d \gamma=0, \\
\sum_{i=1}^{n} \int_{\gamma_{i-1}}^{\gamma_{i}} \exp \left(-k_{i} \gamma\right) \theta_{i \mid \tau=0} d \gamma-\int_{\gamma_{0}}^{\gamma_{n}} t_{0} d \gamma=0 .
\end{gathered}
$$

Substitution of (14) into (21) and (22) taking account of (11), (12) gives

$$
\begin{gathered}
\sum_{i=1}^{n} \frac{\left(\mathrm{L}_{i}+\mu_{i}\right)\left(q_{i} \theta_{i-1}^{+}+r_{i} \theta_{i}^{+}\right)}{\left(k_{i}^{2}+p_{i}^{2}\right) \sin \left(p_{i} \delta_{i}\right)}-\delta T_{e}^{\mu}=0, \\
\sum_{i=1}^{n} \frac{\left(q_{i} \theta_{i-1 \mid \tau=0}^{+}+r_{i} \theta_{i \mid \tau=0}^{+}\right)}{\left(k_{i}^{2}+p_{i}^{2}\right) \sin \left(p_{i} \delta_{i}\right)}-\delta T_{0}=0,
\end{gathered}
$$

where $\quad T_{e}^{\mu}=\frac{1}{\delta} \sum_{i=1}^{n} \mu_{i} \int_{\gamma_{i-1}}^{\gamma_{i}} t_{e} d \gamma, \quad T_{0}=\frac{1}{\delta} \int_{\gamma_{0}}^{\gamma_{n}} t_{0} d \gamma$,

$$
\begin{aligned}
q_{i} & =\exp \left(-k_{i-1} \gamma_{i-1}\right)\left(p_{i} \exp \left(-k_{i} \delta_{i}\right)+k_{i} \sin \left(p_{i} \delta_{i}\right)-p_{i} \cos \left(p_{i} \delta_{i}\right)\right), \\
r_{i} & =\exp \left(-k_{i} \gamma_{i}\right)\left(p_{i} \exp \left(k_{i} \delta_{i}\right)-k_{i} \sin \left(p_{i} \delta_{i}\right)-p_{i} \cos \left(p_{i} \delta_{i}\right)\right),
\end{aligned}
$$

and $\theta_{0}^{+}=\theta_{1}^{-}=t_{b}$ is a supplement auxiliary notation. 
Substituting from (16) expression for $\theta_{i}^{+}=f_{i}^{11} t_{b}+f_{i}^{12}\left(\frac{\partial t}{\partial \gamma}\right)_{b}$ into (23) and (24), we finally obtain

$$
\begin{gathered}
a_{s 1} t_{b}+a_{s 2}\left(\frac{\partial t}{\partial \gamma}\right)_{b}-\delta T_{e}^{\mu}=0 \text { on } G \\
a_{\tau 1} t_{b \mid \tau=0}+a_{\tau 2}\left(\frac{\partial t}{\partial \gamma}\right)_{b \mid \tau=0}-\delta T_{0}=0 \text { on } S,
\end{gathered}
$$

where $\quad\left[a_{s 1}, a_{s 2}\right]^{\mathrm{T}}=\sum_{i=1}^{n} \frac{\left(\mathrm{L}_{i}+\mu_{i}\right)}{\left(k_{i}^{2}+p_{i}^{2}\right) \sin \left(p_{i} \delta_{i}\right)}\left[\begin{array}{cc}f_{i-1}^{11} & f_{i}^{11} \\ f_{i-1}^{12} & f_{i}^{12}\end{array}\right]\left[\begin{array}{l}q_{i} \\ r_{i}\end{array}\right]$,

$$
\left[a_{\tau 1}, a_{\tau 2}\right]^{\mathrm{T}}=\sum_{i=1}^{n} \frac{1}{\left(k_{i}^{2}+p_{i}^{2}\right) \sin \left(p_{i} \delta_{i}\right)}\left[\begin{array}{cc}
f_{i-1}^{11} & f_{i}^{11} \\
f_{i-1}^{12} & f_{i}^{12}
\end{array}\right]\left[\begin{array}{l}
q_{i} \\
r_{i}
\end{array}\right]
$$

and $f_{i}^{11}, f_{i}^{12}$ are elements of the matrix (17) for $i=\overline{1, n}$; and $f_{0}^{11}=1, f_{0}^{12}=0$.

Since relations (19), (25), (26) establish the connection between the boundary value of body temperature $t_{b}$ and its derivative $\left(\frac{\partial t}{\partial \gamma}\right)_{b}$ with given value of environment temperature $t_{e}$, they can be interpreted as generalized boundary conditions which take into account the influence of the multilayer coating on the course of heat process in a body.

\section{Calculation Variants of Generalized Boundary Conditions}

For sufficiently thin coatings, conditions (19), (25), (26) can be simplified by expanding their terms in the series of powers of small $\delta_{i}$ and neglecting the terms containing $\delta_{k}^{m} \delta_{l}^{s}$ with $m+s \geq 2$. The result is

$$
\begin{gathered}
\sum_{i=1}^{n} \tilde{\Delta}_{i} t_{b}-\lambda_{b}^{\gamma}\left(1-2 K+\frac{\mu}{H}\right)\left(\frac{\partial t}{\partial \gamma}\right)_{b}+\mu\left(t_{e}-t_{b}\right)=\Omega \frac{\partial t_{b}}{\partial \tau}, \quad t_{b \mid \tau=0}=T_{0} \quad \text { on } S, \\
\sum_{i=1}^{n}\left(\mathrm{~L}_{i}+\mu_{i}\right) \delta_{i} t_{b}=\delta T_{e}^{\mu} \quad \text { on } \mathrm{G} .
\end{gathered}
$$

Here $\quad \tilde{\Delta}_{i}=\frac{1}{A_{i} B_{i}}\left[\Lambda_{i}^{\alpha} \frac{\partial}{\partial \alpha}\left(\frac{B_{i}}{A_{i}} \frac{\partial}{\partial \alpha}\right)+\Lambda_{i}^{\beta} \frac{\partial}{\partial \beta}\left(\frac{A_{i}}{B_{i}} \frac{\partial}{\partial \beta}\right)\right] ; \quad \Lambda_{i}^{l}=\lambda_{i}^{l} \delta_{i}$ is the reduced thermal conductivity of the $i$ th layer in the direction $l \quad(l=\alpha, \beta, \gamma)$; $\Omega_{i}=\omega_{i} \delta_{i} ; h_{i}=\lambda_{i}^{\gamma} / \delta_{i}$ are the reduced thermal capacity and thermal permeability of 
the $i$ th layer; $\Omega=\sum_{i=1}^{n} \Omega_{i}, K=\sum_{i=1}^{n} k_{i} \delta_{i}, H^{-1}=\sum_{i=1}^{n} h_{i}^{-1}$ are the reduced thermal capacity, reduced curvature and reduced thermal resistance of the whole coating.

In the case when the principal curvatures and coefficients of the first fundamental form of the interfaces between layers can be regarded as approximately equal to the corresponding quantities for the body-coating interface ( $A=A_{i}, B=B_{i}, k=k_{i}, i=\overline{1, n}$ ), conditions (27) become

$$
\begin{aligned}
\widetilde{\Delta} t_{b}-\lambda_{b}^{\gamma}\left(1-2 K+\frac{\mu}{H}\right)\left(\frac{\partial t}{\partial \gamma}\right)_{b}+\mu\left(t_{e}-t_{b}\right) & =\Omega \frac{\partial t_{b}}{\partial \tau}, \quad t_{b \mid \tau=0}=T_{0} \quad \text { on } S, \\
(\mathrm{~L}+\mathrm{M}) t_{b} & =\delta T_{e}^{\mu} \quad \text { on } \mathrm{G},
\end{aligned}
$$

where $\quad \tilde{\Delta}=\frac{1}{A B}\left[\Lambda^{\alpha} \frac{\partial}{\partial \alpha}\left(\frac{B}{A} \frac{\partial}{\partial \alpha}\right)+\Lambda^{\beta} \frac{\partial}{\partial \beta}\left(\frac{A}{B} \frac{\partial}{\partial \beta}\right)\right], \quad \Lambda^{l}=\sum_{i=1}^{n} \Lambda_{i}^{l}, l=\alpha, \beta$

$$
\mathrm{L}=n_{1} \frac{\Lambda^{\alpha}}{A} \frac{\partial}{\partial \alpha}+n_{2} \frac{\Lambda^{\beta}}{B} \frac{\partial}{\partial \beta}, \quad K=k \delta, \quad \mathrm{M}=\sum_{i=1}^{n} \mu_{i} \delta_{i} .
$$

For the cases when the shell that models the coating is closed, conditions (27)-(28) coincide with the ones, which have been obtained in [10]. Such modeling is also possible when the influence of the edge surface can be neglected due to its small value in the whole heat transfer.

\section{Restoration Formulas}

After determining the temperature field in a body based on solving the appropriate boundary value problem with generalized boundary conditions, we can find temperature over the thickness of a coating by restoration formulas.

These formulas can be obtained by substitution of change (6) to (14) using (16), (11), and (12). In a general case, they have the form

$$
\begin{aligned}
t_{i}= & \exp \left(-k_{i} \gamma\right)\left\{\frac{\exp \left[\left(k_{i}-k_{i-1}\right) \gamma_{i-1}\right] \sin \left(p_{i}\left(\gamma_{i}-\gamma\right)\right) f_{i-1}^{11}+\sin \left(p_{i}\left(\gamma-\gamma_{i-1}\right)\right) f_{i}^{11}}{\sin \left(p_{i} \delta_{i}\right)} t_{b}+\right. \\
& \left.+\frac{\exp \left[\left(k_{i}-k_{i-1}\right) \gamma_{i-1}\right] \sin \left(p_{i}\left(\gamma_{i}-\gamma\right)\right) f_{i-1}^{12}+\sin \left(p_{i}\left(\gamma-\gamma_{i-1}\right)\right) f_{i}^{12}}{\sin \left(p_{i} \delta_{i}\right)}\left(\frac{\partial t}{\partial \gamma}\right)_{b}\right\} i=\overline{1, n}
\end{aligned}
$$

Expanding (29) in power series for small $\delta_{i}$, and omitting terms containing $\delta_{k}^{m} \delta_{l}^{s}$ with $m+s \geq 2$, we receive the following calculation variant

$$
t_{i}=t_{b}+\lambda_{b}^{\gamma}\left[\sum_{j=1}^{i-1} \frac{1-2 K_{j-1 / 2}}{h_{j}}+\frac{1-2 K_{i-1 / 2}+k_{i}\left(\gamma_{i}-\gamma\right)}{\lambda_{i}}\left(\gamma-\gamma_{i-1}\right)\right]\left(\frac{\partial t}{\partial \gamma}\right)_{b} i=\overline{1, n}
$$

where $K_{i-1 / 2}=\sum_{j=1}^{i-1} k_{j} \delta_{j}+\frac{k_{i} \delta_{i}}{2}$. 


\section{Test Problem}

The efficiency of suggested approach is illustrated by the comparison of results, obtained according to the above approximate approach with the solution of a test onedimension problem of non-stationary heat conduction when heating the plate with a threelayer isotropic coating.

Equations of heat conduction and initial conditions for the $i$-th layer and the body have the form

$$
\begin{gathered}
\frac{\partial t_{i}}{\partial \tau}=a_{i} \frac{\partial^{2} t_{i}}{\partial x^{2}}, \\
t_{i \mid \tau=0}=0, \quad i=1,2,3, b .
\end{gathered}
$$

We assume that the coating-environment boundary surface is heated according to Newton's law and the opposite side of the plate is thermally insulated

$$
\begin{gathered}
\lambda_{3} \frac{\partial t_{3}}{\partial x}=\mu\left(t_{3}-t_{e}\right) \text { at } x=-\left(\delta_{1}+\delta_{2}+\delta_{3}\right), \\
\frac{\partial t_{b}}{\partial x}=0 \quad \text { at } x=h .
\end{gathered}
$$

The conditions of ideal thermal contact occur at the interfaces

$$
\begin{array}{lll}
t_{3}=t_{2}, & \lambda_{3} \frac{\partial t_{3}}{\partial x}=\lambda_{2} \frac{\partial t_{2}}{\partial x} & \text { at } x=-\left(\delta_{1}+\delta_{2}\right) ; \\
t_{2}=t_{1}, & \lambda_{2} \frac{\partial t_{2}}{\partial x}=\lambda_{1} \frac{\partial t_{1}}{\partial x} & \text { at } x=-\delta_{1} ; \\
t_{1}=t_{b}, & \lambda_{1} \frac{\partial t_{3}}{\partial x}=\lambda_{b} \frac{\partial t_{2}}{\partial x} & \text { at } x=0 .
\end{array}
$$

Here $a_{i}=\lambda_{i} / \omega_{i}$ is the coefficient of thermal diffusivity of the $i$ th layer $(i=1,2,3)$ and of the body $(i=b) ; h$ is the thickness of the body.

The exact solution of this problem is given in [13]. The approximate solution of the problem on the basis of the above formulated approach is based on the solution of equation (31) in the body domain under the initial condition (32), the boundary condition of insulation (34), and the generalized boundary condition following from (28), which in the considered case has the form

$$
\begin{gathered}
\lambda_{b}\left(1+\frac{\mu}{H}\right) \frac{\partial t_{b}}{\partial x}+\mu\left(t_{e}-t_{b}\right)=\Omega \frac{\partial t_{b}}{\partial \tau}, \quad t_{b \mid \tau=0}=0 \quad \text { at } x=0 ; \\
\frac{1}{H}=\frac{\delta_{1}}{\lambda_{1}}+\frac{\delta_{2}}{\lambda_{2}}+\frac{\delta_{3}}{\lambda_{3}}, \quad \Omega=\omega_{1} \delta_{1}+\omega_{2} \delta_{2}+\omega_{3} \delta_{3} .
\end{gathered}
$$

Using the Laplace transformation, we represent the solution of this problem in the form 


$$
\begin{gathered}
\frac{t_{b}(x, \tau)}{t_{e}}=1-\sum_{j=1}^{\infty} \frac{2 \mu h^{2} U\left(x, \kappa_{j}\right) \exp \left(-\kappa_{j}^{2} a_{b} \tau\right)}{Z\left(\kappa_{j}\right)}, \quad 0 \leq x \leq h, \\
U\left(x, \kappa_{j}\right)=\cos \kappa_{j} \cos \frac{x \kappa_{j}}{h}+\sin \kappa_{j} \sin \frac{x \kappa_{j}}{h}, \\
Z\left(\kappa_{j}\right)=\left[2 \Omega a_{b}+\lambda_{b}\left(1+\frac{\mu}{H}\right) h\right] \kappa_{j} \cos \kappa_{j}+\left[\mu h^{2}-\Omega a_{b} \kappa_{j}^{2}+\lambda_{b}\left(1+\frac{\mu}{H}\right) h\right] \sin \kappa_{j}, \\
\text { where } \kappa_{j} \text { are roots of the equation } \operatorname{tg\kappa }=\frac{\left(\mu h^{2}-\Omega a_{b} \kappa\right)}{\lambda_{b}\left(1+\frac{\mu}{H}\right) h \kappa} .
\end{gathered}
$$

Substituting (37) in the restoration formulas (30), we obtain the expression for temperature in the coating:

$$
\begin{gathered}
\frac{t_{i}(x, \tau)}{t_{e}}=1-\sum_{j=1}^{\infty} \frac{2 \mu h^{2} V\left(x, \kappa_{j}\right) \exp \left(-\kappa_{j}^{2} a_{b} \tau\right)}{Z\left(\kappa_{j}\right)}, \quad-\left(\delta_{1}+\delta_{2}+\delta_{3}\right) \leq x \leq 0, \\
V\left(x, \kappa_{j}\right)=\cos \kappa_{j}+\lambda_{b}\left(\sum_{j=1}^{i-1} h_{j}^{-1}+\frac{x-\gamma_{i-1}}{\lambda_{i}}\right) \kappa_{j} \sin \kappa_{j} .
\end{gathered}
$$

This approximate solution is compared with exact solution according [13] for certain time moments for the four-layer plate characterized by the following values of geometrical and physical parameters: $\delta_{1}=\delta_{2}=0.5 \delta_{3}=5 \cdot 10^{-5} \mathrm{~m}, \quad h=2 \cdot 10^{-2} \mathrm{~m}$, $\lambda_{1}=2 \mathrm{~W} / \mathrm{m} \cdot{ }^{0} \mathrm{C}, \quad \lambda_{2}=4 \mathrm{~W} / \mathrm{m} \cdot{ }^{0} \mathrm{C}, \quad \lambda_{3}=6 \mathrm{~W} / \mathrm{m} \cdot{ }^{0} \mathrm{C}, \quad \lambda_{b}=4 \mathrm{~W} / \mathrm{m} \cdot{ }^{0} \mathrm{C}$, $a_{1}=5 \cdot 10^{-6} \mathrm{~m}^{2} / \mathrm{S}, \quad a_{2}=6.4 \cdot 10^{-6} \mathrm{~m}^{2} / \mathrm{S}, \quad a_{3}=8 \cdot 10^{-6} \mathrm{~m}^{2} / \mathrm{S}, \quad a_{b}=1.2 \cdot 10^{-5} \mathrm{~m}^{2} / \mathrm{S}$, $t_{e}=100{ }^{0} C, \quad B i_{1}=\mu\left(\delta_{1}+\delta_{2}+\delta_{3}\right) / \lambda_{1}=1$.

The analysis of the indicated results obtained for certain time moments (Table 1) shows that the values of temperatures of exact (lower values in the lines) and approximate (upper values in the lines) solutions do not differ essentially.

Table 1. Comparison of exact results and approximate solution

\begin{tabular}{|c|c|c|c|c|c|c|c|c|}
\hline \multirow{2}{*}{$\begin{array}{c}\tau \\
\text { sec }\end{array}$} & $x=-\gamma_{3}$ & $x=-\gamma_{2}$ & $x=-\gamma_{1}$ & $x=0$ & $x=0.25 h$ & $x=0.5 h$ & $x=0.75 h$ & $x=h$ \\
\cline { 2 - 9 } & 12.040 & 11.901 & 11.796 & 11.587 & 3.991 & 0.955 & 0.153 & 0.031 \\
\multirow{2}{*}{2} & 12.047 & 11.830 & 11.722 & 11.581 & 3.987 & 0.953 & 0.152 & 0.032 \\
\hline \multirow{2}{*}{10} & 24.455 & 24.333 & 24.241 & 24.057 & 16.015 & 10.320 & 6.959 & 5.852 \\
& 24.458 & 24.271 & 24.178 & 24.055 & 16.013 & 10.319 & 6.960 & 5.856 \\
\hline \multirow{2}{*}{40} & 48.287 & 48.203 & 48.140 & 48.014 & 42.417 & 38.312 & 35.806 & 34.963 \\
& 48.294 & 48.166 & 48.102 & 48.018 & 42.422 & 38.318 & 36.813 & 34.972 \\
\hline \multirow{2}{*}{100} & 75.522 & 75.483 & 75.453 & 75.393 & 72.744 & 70.801 & 69.615 & 69.216 \\
& 75.531 & 75.470 & 75.440 & 75.400 & 72.752 & 70.810 & 69.624 & 69.226 \\
\hline \multirow{2}{*}{500} & 99.833 & 99.833 & 99.832 & 99.832 & 99.814 & 99.801 & 99.792 & 99.790 \\
& 99.833 & 99.833 & 99.832 & 99.832 & 99.814 & 99.801 & 99.793 & 99.790 \\
\hline
\end{tabular}




\section{Concluding Remarks}

These generalized boundary conditions may be used for an analytical solution (when it is possible) as well as for a numerical one. Since the application of direct numerical methods without the preliminary transformation of initial problems for massive bodies with thin coatings may be impeded (because of the essential difference between geometrical and thermal characteristics of the coating and the base), one may expect the efficiency of application of approaches based on the use of generalized boundary conditions. The suggested approach has the following advantages:

1. It allows us, on the basis of a generalized model, to simplify essentially the calculation of constructions with thin multilayer coatings and to reduce, respectively, the time taken by computation.

2. It gives a possibility of obtaining in certain cases relatively simple analytical solutions of important practical problems for piecewise homogeneous media, which allow us to provide a priori qualitative and quantitative estimation of the thermal state of the constructions without cumbersome calculations.

3. The efficiency of this approach increases with the decrease of the coating thickness in contrast to the application of direct methods without preliminary transformation of initial problems.

\section{References}

1. Al Nimr, M.A., Alcam, M.K.: A generalized thermal boundary condition. Int. J. Heat and Mass Transfer. 33 (1997) 157-161

2. Elperin, T., Rudin, G.: Analytical solution of heat conduction problem for a multilayer assembly arising in photothermal reliability testing. Int. Comm. in Heat and Mass Transfer. 21 (1994) 95-104

3. Fleishman, N.P.: Mathematical models of thermal conjugation of media with thin foreign insertions or coatings. Visnyk of L'viv University. Ser. Mech-Math. 39 (1993) 30-34

4. Podstrigach, Ya.S.: On application of operator method to derivation of main relations of theory of heat conduction in thin-walled construction elements. Teplovye Napryazhenia $\mathrm{V}$ Elementakh Konstruktsij (Thermal Stresses in Construction Elements). 5 (1965) 24-35

5. Podstrigach, Ya.S., Shevchuk, P.R.: Temperature fields and stresses in bodies with thin coverings. Ibid. 7 (1967) 227-233

6. Podstrigach, Ya.S., Shevchuk, P.R., Onufrik T.M., Povstenko Yu.Z.: Surface effects in solid bodies taking into account coupling physico-mechanical processes. Physico-chemical Mechanics of Materials. 11 (1975) 36-41

7. Podstrygach, Ya.S., Shvets, R.N.: Thermoelasticity of Thin Shells. Naukova Dumka, Kiev (1978)

8. Savula, Ya.H.: Mathematical model of heat conduction through three-dimensional body with thin plate coating. Visnyk of L'viv University. Ser. Mech-Math. 42 (1995) 3-7

9. Savula, Ya.H., Dyyak, I. I., Krevs, V.V.: Heterogeneous mathematical models in numerical analysis of structures. Computers and Mathematics with Applications. 42 (2001) 1201-1216

10.Shevchuk V.A.: Generalized boundary conditions for heat transfer between a body and the surrounding medium through a multilayer thin covering. J. Math. Sci. 81 (1996) 3099-3102

11.Shvets, Yu.I, Prokopov, V.G., Fialko, N. M., et al.: Thermal state of parts with multilayer lacquer coatings. Visnyk of Academy of Sciences of Ukraine. 5 (1987) 39-44

12.Tret'yatchenko, G.N., Barilo, V.G.: Thermal and stressed state of multilayer coatings. Problems of Strength. 1 (1993) 41-43

13.Vendin, S.V.: On calculation of non-stationary heat conduction in multilayer objects with boundary conditions of the third kind. J. Eng. Physics and Thermophysics 65 (1994) 823-825 